Article

\title{
Novel High-Power, High Repetition Rate Laser Diode Pump Modules Suitable for High-Energy Class Laser Facilities
}

\author{
Marko Hübner ${ }^{1, *}$, Ingo Will ${ }^{2}$, Jörg Körner ${ }^{3}{ }^{(0)}$, Jürgen Reiter ${ }^{3}$, Mathias Lenski ${ }^{3}$, \\ Johannes Tümmler ${ }^{2}$, Joachim Hein ${ }^{3}$ DD, Bernd Eppich ${ }^{1}$, Arnim Ginolas ${ }^{1}$ and Paul Crump ${ }^{1}$ \\ 1 Ferdinand-Braun-Institut, Leibniz-Institut für Höchstfrequenztechnik (FBH), Gustav-Kirchhoff-Str. 4, \\ 12489 Berlin, Germany \\ 2 Max-Born-Institut für Nichtlineare Optik und Kurzzeitspektroskopie (MBI), Max-Born-Straße 2 A, \\ 12489 Berlin, Germany \\ 3 Institute for Optics and Quantum Electronics (IOQ), Friedrich-Schiller University Jena, Max-Wien-Platz 1, \\ 07743 Jena, Germany \\ * Correspondence: marko.huebner@fbh-berlin.de; Tel.: +49-30-6392-2716
}

Received: 29 May 2019; Accepted: 24 June 2019; Published: 4 July 2019

\begin{abstract}
The latest generation of high-energy-class pulsed laser facilities, under construction or planned, such as EuPRAXIA, require reliable pump sources with high power (many $\mathrm{kW}$ ), brightness $\left(>1 \mathrm{MW} / \mathrm{cm}^{2} / \mathrm{sr}\right.$ ) and electro-optical conversion efficiency $(>50 \%)$. These new facilities will be operated at high repetition rates (around $100 \mathrm{~Hz}$ ) and only diode lasers are capable of delivering the necessary performance. Commercial (quasi-continuous wave, QCW) diode laser pulse-pump sources are, however, constructed as low-cost passively cooled stacked arrays that are limited either in brightness, efficiency or repetition rate. Commercial continuous wave diode laser pumps constructed using microchannel coolers (as used in high-value industrial machine tools) can fulfil all requirements, but are typically not preferred, due to their cost and complexity and the challenges of preventing cooler degradation. A custom solution is shown here to fill this gap, using advanced diode lasers in a novel passive side-cooling geometry to realize $100 \ldots 200 \mathrm{~Hz}$. pump modules (10\%-20\% duty cycle) that emit peak power of $6 \mathrm{~kW}$ at wavelength $=940 \mathrm{~nm}$. The latest performance of these modules is summarized and compared to literature. We show that a brightness $>1 \mathrm{MW} / \mathrm{cm}^{2} / \mathrm{sr}$ can be efficiently delivered across a wide range of laser pulse conditions with $10 \%$ duty cycle (pulse width: $100 \mu$ s... $100 \mathrm{~ms}$... cw, repetition rate up to $1 \mathrm{kHz}$ ). Furthermore, we describe how these pumps have been used to construct and reliably operate $\left(>10^{9}\right.$ pulses without degradation) in high-energy-class regenerative and ring amplifiers at the Max-Born-Institut für Nichtlineare Optik und Kurzzeitspektroskopie (MBI). We also show first results on $100 \mathrm{~Hz}$ pumping of cryogenically cooled solid-state Yb:YAG slab amplifiers, as anticipated for use in the EuPRAXIA laser, and note that peak temperature is disproportionately increased, indicating that improved cooling and more detailed studies are needed.
\end{abstract}

Keywords: diode laser; diode stacks; $100 \mathrm{~Hz}$ pumping; high duty cycle; fibre coupled; EuPRAXIA

\section{Introduction}

EuPRAXIA (European Plasma Research Accelerator with Excellence in Applications) is a consortium that works on a conceptual design study for the world's first $5 \mathrm{GeV}$ particle accelerator with industry-ready beam quality and a pulse repetition rate of $100 \mathrm{~Hz}$ [1]. Unlike most conventional accelerators, the new design utilises strong field gradients in a gas or plasma which are created by a particle beam or a high-power laser pulse. These fields, so called wakefields, can reach field strengths that are 1000 times stronger than in conventional accelerators and, therefore, result in more highly 
energised particles. Intense, efficient diode laser pump sources are particularly important for these systems, and these sources generate all the photons in the system, as discussed in [1]. The exact pump requirements will depend on the final system design. One strong candidate for the system design of the highest power EuPRAXIA laser ("Laser 3") makes use of a Ti-sapphire laser, itself pumped by cryogenically cooled Yb:YAG power amplifier, derived from the DIPOLE100 laser [2-4], that operates with $f=10 \mathrm{~Hz}$. Initial estimates indicate that appropriate diode laser pump sources for the scaled DIPOLE100 would need to emit peak powers of $P_{\text {opt }}=300 \mathrm{~kW}$ to $1200 \mathrm{~kW}$ at a brightness of $B>1 \mathrm{MW} / \mathrm{cm}^{2} / \mathrm{sr}$ (with $B$ defined as in [5] in terms of $P_{\text {opt }}$ emitted into a solid angle), with emission wavelength $\lambda=940 \mathrm{~nm}$, pulse length $\tau_{\mathrm{p}}=0.5 \ldots 2 \mathrm{~ms}$ and increased repetition rates of $f=20 \ldots$ $100 \mathrm{~Hz}$. Such large-scale sources have a significant production cost, which can be estimated based on published values for commercial system build, on a $€ / W$ basis (see e.g., [6]). Commercially available pump sources that can potentially deliver these parameters consist of stacks or arrays of high-energy class diode lasers (HEC-DL) in 1-cm bar format, with each bar combined with a fast axis collimation (FAC) lens. The bars are collected into pump modules, including customised optics for beam shaping and homogenizing [7-12]. There are two main configurations. First, laser bars are assembled into economically cooled pulsed-current stacks, where the bars are densely packed and cooled from their rear edges, either passively or in modules with integrated large-core water channels (also at the rear edges). These packages have limited cooling power, and are restricted to low duty cycle operation, or limited output power and hence brightness $B$ and are widely used in signalling, sensing, military (e.g., targeting) and medical (e.g., dermatology) applications. Second, laser bars are assembled using microchannel coolers, which incorporate thin $(\sim 10-50 \mu \mathrm{m})$ water channels directly below the lasers bars, for efficient heat extraction. The effective cooling allows these units to operate from pulsed up to continuous wave conditions at high $P_{\text {opt }}$, and they are in wide use in high value-add laser material processing (e.g., metal cutting) systems. However, microchannel coolers are often not preferred for large systems such as EuPRAXIA, due to fabrication cost and challenges in control of the water for long lifetimes (due to erosion, corrosion, deposition effects).

Table 1 collects the recently published performance of commercial pump modules that are relevant for the EuPRAXIA project. As one can see, these modules were operated with parameters that, to some extent, overlap with those required. For example, some publications focus on other wavelengths than $=940 \mathrm{~nm}$ (e.g., =808 nm), but these modules can very likely be modified for similar performance at $940 \mathrm{~nm}$. Also shown is the optical brightness $B$ of the pump, where the emission aperture was either directly taken from the reference or calculated by the authors as in [8] from the emitting area (integrated over all emitters in the module). The solid angle was also calculated following [5], using the lateral and vertical divergences angles with $95 \%$ power content. If no fast angle was given, the authors assumed an angle after collimation of $0.36^{\circ}$. Table 1 lists also the achievable values for pulse width and repetition rate, which are highly dependent on the cooling capability of the package used. Typical pulse widths lay in the $0.2 \ldots 400 \mathrm{~ms}$ range with pulse repetition ranging from about $1 \mathrm{~Hz}$ to $400 \mathrm{~Hz}$.

Overall, commercially available pump sources partly fulfil the EuPRAXIA requirements. Some achieve the requested brightness of $>1 \mathrm{MW} / \mathrm{cm}^{2} / \mathrm{sr}[2,5,7,9,13]$ others fulfil the required $f=20 \mathrm{~Hz}$ to $100 \mathrm{~Hz}$ [7,9]. Full performance is only achieved when microchannel coolers are allowed. Table 1 also shows the performance of an alternative HEC-DL pump design, developed and used in high-energy class pump applications by the authors, which offers high $P_{\mathrm{opt}}, B, f$ and $\tau_{\mathrm{p}}$ without the need for microchannel cooling. The high performance is enabled though the use of novel chip, submount and stack design, as well as the use of robust high-power diode laser technology. The approach was described in detail in [14] and key points are summarized in Section 2. 
Table 1. Performance parameters of high-energy class diode laser (HEC-DL) pulsed pump modules compared to expected requirements for large scale high-repetition rate systems such as EuPRAXIA (numbers in bold show where there is a match to requirements).

\begin{tabular}{|c|c|c|c|c|c|c|c|}
\hline Source & $\begin{array}{l}\text { Aperture } \\
{\left[\mathrm{cm}^{2}\right]}\end{array}$ & $\begin{array}{l}\text { Solid Angle } \\
\text { [sr] }\end{array}$ & $\begin{array}{c}\text { Peak Optical Power } P_{\text {opt }} \\
{[\mathrm{kW}]}\end{array}$ & $\begin{array}{l}\text { Brightness } B \\
{\left[\mathrm{MW} / \mathrm{cm}^{2} / \mathrm{sr}\right]}\end{array}$ & $\begin{array}{c}\text { Repetition Rate, } f \\
{[\mathrm{~Hz}]}\end{array}$ & $\begin{array}{l}\text { Pulse Width, } \tau_{\mathrm{p}} \\
{[\mathrm{ms}]}\end{array}$ & $\begin{array}{l}\text { Wavelength } \\
{[\mathrm{nm}]}\end{array}$ \\
\hline \multicolumn{8}{|c|}{ Anticipated pump requirement for EuPRAXIA “Laser 3” } \\
\hline & & & $300-1200$ & $0.8-1.0$ & $20 . .100$ & $0.5 \ldots 2$ & 940 \\
\hline \multicolumn{8}{|c|}{ Results here } \\
\hline $\mathrm{FBH}^{*}$, Stack & 1.1 & 0.00131 & 3.6 & 2.57 & $1000 \ldots 1$ & $\begin{array}{c}0.1 \ldots 100 \ldots \\
(\mathrm{cw})\end{array}$ & 940 \\
\hline FBH, Module & 1.1 & 0.00131 & 6 & 4.29 & $1000 \ldots 1$ & $\begin{array}{c}0.1 \ldots 100 \ldots \\
(\mathrm{cw})\end{array}$ & 940 \\
\hline \multicolumn{8}{|c|}{ Other publications } \\
\hline [5], Stack & 1.3 & 0.00120 & 2.2 & 1.41 & 10 & 1 & $808 / 940$ \\
\hline [5], Module & 4.0 & 0.01065 & 20 & 0.47 & 10 & 1 & 940 \\
\hline [10], 8 bars & 1.2 & 0.00120 & 0.5 & 0.36 & $1.375 / 3$ & $400 / 50$ & 810 \\
\hline [10], 12 bars & 1.3 & 0.00120 & 1.08 & 0.68 & 2 & 100 & 810 \\
\hline$[7]^{\text {con }}$ & 1.2 & 0.00120 & 4.4 & 3.07 & 10 & 1.2 & 980 (“766-1550”) \\
\hline [7] in & 1.9 & 0.00120 & 1.32 & 0.59 & $1.375 / 3 / 400$ & $400 / 50 / 0.2$ & 980 (“766-1550”) \\
\hline [7], Module mcc & 111.6 & 0.00120 & 250 & 1.86 & 400 & 0.2 & 808 \\
\hline [9], 3 bars & 0.2 & 0.00120 & 0.7 & 2.91 & 50 & 0.25 & 808 \\
\hline [9], 5 bars & 0.4 & 0.00120 & 1.5 & 3.11 & 20 & 0.25 & 808 \\
\hline [13], 8 bars & 1.3 & 0.00106 & 4.8 & 3.58 & 10 & 1 & 935 \\
\hline [2], DiPOLE100 & 62.4 & 0.00381 & 250 & 1.05 & 10 & 1 & 940 \\
\hline$[15], 25$ bars & 4.3 & 0.00094 & 12 & 3.07 & 10 & 1 & 938 (“880-1000") \\
\hline
\end{tabular}

* Ferdinand-Braun-Institute, ${ }^{\text {con }}$ conductively cooled, ${ }^{\text {in }}$ indirectly cooled ${ }^{\mathrm{mcc}}$ microchannel cooled. 
Following this brief introduction, the article is structured as follows. Firstly, a short summary is given of the authors' approach for high duty cycle HEC-DL pump sources, covering design, electrical, optical and spectral characteristics and beam quality. Secondly, two examples of successful room temperature application are given, as pumps for disk amplifiers. Finally, results of initial high repetition rate pump experiments of cryogenically cooled Yb:YAG slabs are discussed, before concluding.

\section{Materials and Methods}

\subsection{Module Design}

Due to the absence of high repetition rate, micro-channel-free packaging solutions for HEC-DLs, an alternative configuration was developed, as described in detail in [12] and repeated here for convenience. The module design is based on the geometrical beam combination of wide-aperture $(1200 \mu \mathrm{m})$ single emitters instead of bars. These emitters make use of an "extreme double asymmetric (EDAS) (Patents: EDAS: US Patent No. US8798109, EU Patent No. EP2702694; Passivation: German Patent No. DE10221952)" (vertical) epitaxial design, tailored for high conversion efficiency at high bias, and are processed using standard techniques into diode lasers with cavity length of $6 \mathrm{~mm}$. Facet passivation $^{1}$ ensures the high reliability of the chips at high optical output and the front and rear facets are subsequently AR and HR coated, respectively. The resulting emitters are hard-soldered junction down using AuSn between specifically designed expansion-matched CuW heat spreaders, sandwiching the chip and ensuring good heat removal. At this point, each laser is characterized up to the targeted operation power of $P_{\text {opt }}=120 \mathrm{~W}$ (including a 1-week long burn-in at $P_{\text {opt }}$ ), well below the determined catastrophic optical damage (COD) level of $240 \mathrm{~W}$ [14], reaching a power conversion efficiency at $P_{\text {opt }}$ of $\eta_{\mathrm{E}}=50 \% \ldots 60 \%$ (before fibre). The operating power is equivalent to around $1 \mathrm{~kW}$ from a 1-cm laser bar (with failure power $>2 \mathrm{~kW}$ per bar), corresponding to at least a factor of two higher brightness directly from the semiconductor than available from commercial bar-based solutions. Soldering 28 of these mounted single emitters on top of each other allows stacks with a nominal output power of $3.5 \mathrm{~kW}$ to be realised. Heat removal from the single stack elements is achieved by laterally soldering water-cooled direct copper bonding (DCB) coolers to the stack and extracting heat from the edges of the coolers. The surface area for heat extraction for these $P_{\mathrm{opt}}=3.5 \mathrm{~kW}$ sources is around $6-7 \times$ larger $\left(10 \mathrm{~cm}^{2}\right.$, from 28 emitters on $3 \mathrm{~mm}$ vertical pitch, cooled on two sides along $6 \mathrm{~mm}$ resonator) than in conventional rear-edge-cooled bar stacks (for example, $1.2 \mathrm{~cm}^{2}$, from an 8 bar stack emitting $P_{\text {opt }} \sim 4.4 \mathrm{~kW}$ with a $1.7 \ldots 2 \mathrm{~mm}$ vertical pitch [7], cooled along the 1-cm wide rear edge). The more effective heat spreading and high heat capacity due to larger volume of metal enables higher duty cycles and longer pulses without compromising $B$ or $\eta_{\mathrm{E}}$. A FAC lens is positioned in front of each single stack element for vertical collimation. The complete pump module features two of these stacks whose beams are geometrically combined, first by laterally focusing each stack with a common acylindrical lens through a prism stack consisting of thin right-angle prims. By deflecting the beam of one stack and letting the beam of the other pass, it interleaves both for higher intensity. The final combined beam is focused into a $1.9 \mathrm{~mm}$ diameter spot via a cylindrical (lateral) and an acylindrical (vertical) lens, and collected by a $1.9 \mathrm{~mm}$ core diameter fibre (with NA of 0.22 ) for ex-fibre powers of 5 .. $6 \mathrm{~kW}$ and an efficiency of $\sim 50 \%$ for the whole module. Mechanically, the completed pump module is robustly mounted onto a base plate of dimensions $600 \times 380 \mathrm{~mm}$ allowing its flexible and easy setup for pumping applications. The performance characteristics of the module and stacks is reviewed in the sections below. 


\subsection{Optical and Electrical Power}

The electro-optical characteristics of a module are given in Figure 1. The graph shows the optical pulse power vs. the driving current for a pulse width of $\tau_{p}=1000 \mu$ s and a pulse repetition frequency of $f=100 \mathrm{~Hz}$. That duty cycle, d.c., was chosen because of its relevance for the EuPRAXIA consortium but other combinations of pulse parameters (current, pulse width and repetition frequency) and even continuous wave $(\mathrm{cw})$ operation are also possible. Pulse widths in the range $\tau_{\mathrm{p}}=100 \mu \mathrm{s} \ldots 100 \mathrm{~ms}$ at d.c. $=10 \%$ corresponding to $f=1 \mathrm{kHz} \ldots 1 \mathrm{~Hz}$ have been routinely applied in the lab. The optical pulse power varies close-to linearly with the driver current and, for the module shown, reaches $6 \mathrm{~kW}$ at a current of $140 \mathrm{~A}$ which corresponds to pulse energy of $6 \mathrm{~J}$ per pulse ex-fibre. The beam emitted from the $1.9 \mathrm{~mm}$ core fibre has a brightness $B=1.15 \mathrm{MW} / \mathrm{cm}^{2} / \mathrm{sr}$. The highest electro-optical conversion efficiency $\eta_{E}$ of $50 \%$ ex-fibre is achieved at around $90 \mathrm{~A}$ and remains $\sim 50 \%$ (ex-fibre) up to the highest assessed nominal current of $140 \mathrm{~A}$. These modules were delivered to colleagues from the Max-Born-Institute Berlin, see Section 3, and have been in constant use since then. Figure 1 compares the module's pulse power measured at Ferdinand-Braun-Institut, Leibniz-Institut für Höchstfrequenztechnik (FBH) with those independently measured at Max-Born-Institute. Each module was operated in quasi-continuous wave (QCW) mode with $\tau_{\mathrm{p}}=1 \mathrm{~ms}$ and $f=100 \ldots 200 \mathrm{~Hz}$ with a cooling water temperature of $17^{\circ} \mathrm{C}$ and they all reach powers between 5.4 and $6 \mathrm{~kW}$.
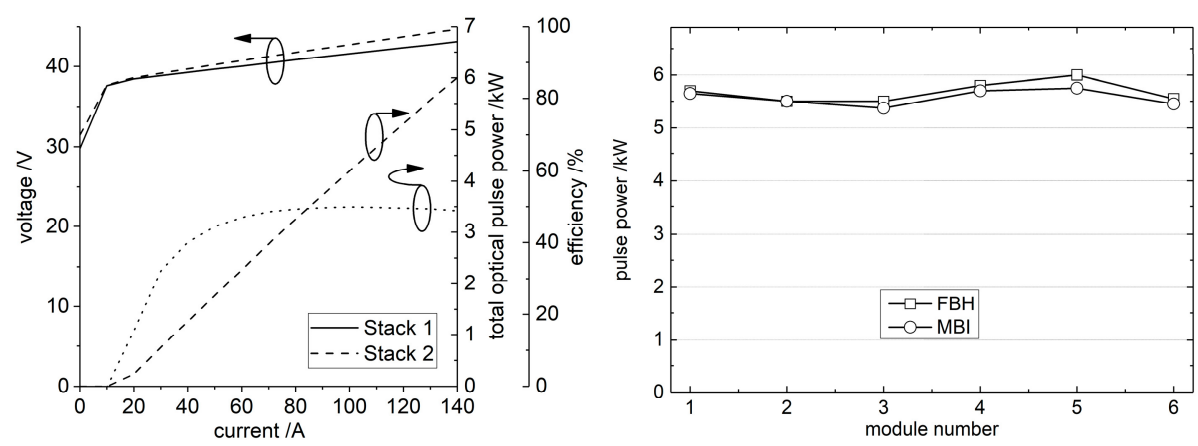

Figure 1. Left: Bias voltage (for the two stacks in a module), optical power and power conversion efficiency as a function of drive current for a Ferdinand-Braun-Institut, Leibniz-Institut für Höchstfrequenztechnik (FBH) pump module $\left(\tau_{\mathrm{p}}=1 \mathrm{~ms}, f=100 \mathrm{~Hz}, \mathrm{FBH}\right.$ data). Right: Ex-fiber operating power as a function of module number for a series of six delivered HEC-DL pump modules $\left(\tau_{\mathrm{p}}=1 \mathrm{~ms}, f=100 \mathrm{~Hz}, \mathrm{FBH}\right.$ and Max-Born-Institut für Nichtlineare Optik und Kurzzeitspektroskopie (MBI) data) [12].

\subsection{Spectral Characteristics}

Typical spectral characteristics for an FBH HEC-DL pump module for various driving regimes are shown in Figure 2. The duty cycle is kept constant at d.c. $=10 \%$, and spectra for pulse repetitions of $f=1 \mathrm{~Hz}, 10 \mathrm{~Hz}, 100 \mathrm{~Hz}$ and $1000 \mathrm{~Hz}$ are shown. The centre wavelength $\lambda_{\mathrm{c}}$ shifts from $937.9 \mathrm{~nm}$ $(f=1000 \mathrm{~Hz})$ to $945.6 \mathrm{~nm}(f=1 \mathrm{~Hz})$, corresponding to a spectral shift of just $8 \mathrm{~nm}$ for 3 orders of magnitude in $f$. We note that a spectral shift of just $18 \mathrm{~nm}$ is seen even for $\mathrm{cw}$ operation, showing the excellent heat dissipation of the stack design. The spectral width $\Delta \lambda_{95 \%}$ ( $95 \%$ power content) is also shown in Figure 2 for the pulsed and cw operation. Under pulsed operation, the spectral width increases from $5 \mathrm{~nm}$ close to threshold up to $18 \mathrm{~nm}$ at $P_{\text {opt }}$ for the $\tau_{\mathrm{p}}=100 \mathrm{~ms}$. In cw mode $\Delta \lambda_{95 \%}$ is $7 \mathrm{~nm}$ (at $20 \mathrm{~A}$ ) and increases up to $26 \mathrm{~nm}$ for the highest tested current, i.e., $80 \mathrm{~A}$. Both $\Delta \lambda_{95 \%}$ and $\lambda_{\mathrm{c}}$ are in ranges suitable for pumping gain media such as Yb:YAG, Yb:LuAG and Yb:CaF 2 [16]. 

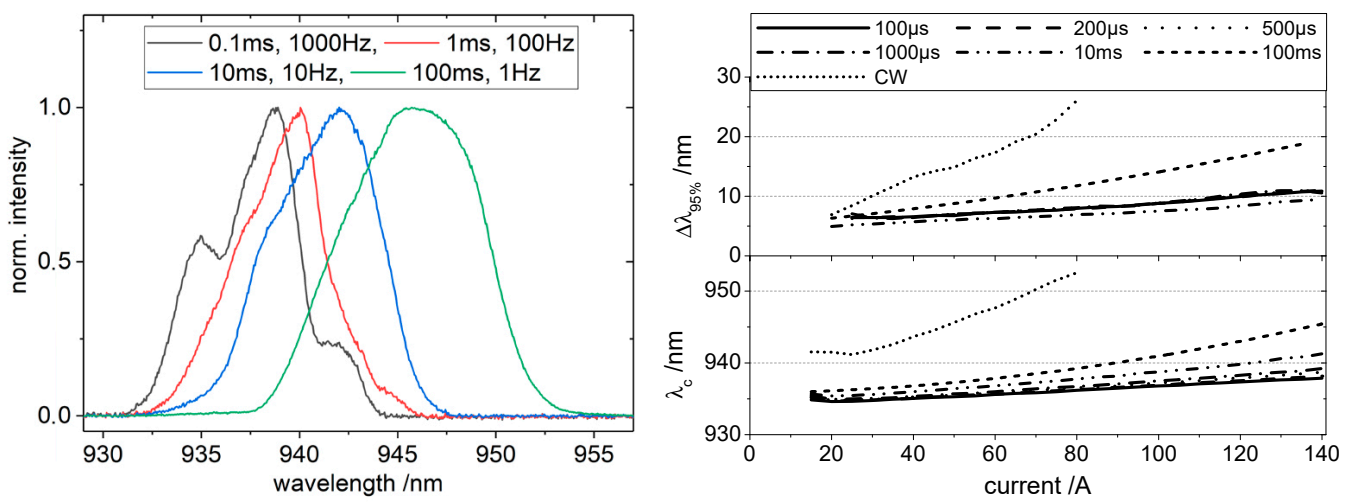

Figure 2. Spectral characteristics for various pulse widths and repetition rates at d.c. $=10 \%$ and in $\mathrm{cw}$ mode with heat sink temperature of $17^{\circ} \mathrm{C}$. Left: Normalised spatially integrated optical power from fibre as a function of wavelength (pump module) at a drive current of 140 A. Right: Spectral width $\Delta \lambda_{95 \%}$ with $95 \%$ power content (top) and centroid wavelength $\lambda_{c}$ (bottom) as a function of drive current.

\subsection{Beam Quality Properties}

The beam propagation factor $\mathrm{M}^{2}$ of the geometrically combined beam was also evaluated by imaging the development of the beam waist around the focal plane along the propagation axis with all optical components in place except the fibre. A sample of the beam was imaged into a high resolution infrared (IR)-sensitive camera, which was protected from damage and saturation effects by placing a high reflectivity mirror in the beam path that directed $99 \%$ of $P_{\text {opt }}$ into an isolating beam dump. The camera was moved along the direction of beam propagation ( $z$ direction) around the focal plane, using a motorized stage. The $\mathrm{M}^{2}$ values were calculated by a fit to the measured development of the beam waist with $\mathrm{z}$, following [17]. Figure 3 shows the beam diameter as a function of $\mathrm{z}$ from which $\mathrm{M}^{2}$ was calculated, in lateral (horizontal, $90^{\circ}$ to stack, parallel to emission from the diode lasers) and vertical (fast-axis) directions. All diameters were obtained at the $95 \%$ power content level.
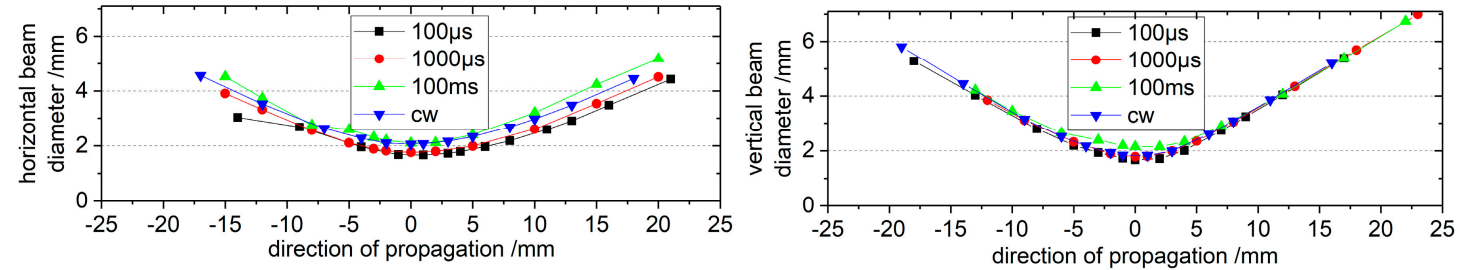

Figure 3. Measured beam diameter as a function of position along the optical (z) axis around the focal plane (caustic) from which the beam quality factor $\mathrm{M}^{2}$ was derived (fit not shown). Measurements were performed at drive current of $140 \mathrm{~A}$ for $100 \mu \mathrm{s}, 1000 \mu \mathrm{s}$ and $100 \mathrm{~ms}$ test and $80 \mathrm{~A}$ for cw with heat sink temperature of $17^{\circ} \mathrm{C}$.

The horizontal beam waist increases with pulse width, whilst the vertical beam diameter also increases with pulse width, but the differences are smaller. Broad area lasers show increases in the horizontal beam diameter with increasing current-driven heating in the laser stripe due to thermal lensing [18,19]. Local heating in the fast-axis collimating micro lenses may also play a role [20]. The lateral and vertical $\mathrm{M}^{2}$ values for various pulse conditions which were calculated from the caustic measurements, Figure 3, are shown in Table 2. As one can see $\mathrm{M}^{2}$ for the horizontal direction varies from 283 to 428 and for the vertical direction varies from 409 to 491 , with cw results in both cases being slightly improved. In our previous studies [12], a stronger trend was seen with pulse width in the vertical beam quality, and this may be due to differences in the quality of the collimation. Further studies are clearly needed. Nevertheless, the measured beam properties values in all directions for all pulse regimes remain around half that of the beam acceptance of the $1.9 \mathrm{~mm}$ core fibre $\left(\mathrm{M}^{2} \sim 700\right)$. 
Therefore, efficient fibre coupling into a narrower fibre for increased brightness should be possible. Coupling into a $1 \mathrm{~mm}$ core diameter fibre $\left(\mathrm{M}^{2}=440\right)$ should also be possible.

Table 2. Beam quality factor $\mathrm{M}^{2}$ for the pump module for various pulse widths and $\mathrm{cw}$ in horizontal and vertical direction, obtained from fitting to the data in Figure 3.

\begin{tabular}{ccc}
\hline Mode of Operation, Duty Cycle $\mathbf{= 1 0} \%$ & $\mathbf{M}^{\mathbf{2}}$, Horizontally & $\mathbf{M}^{\mathbf{2}}$, Vertically \\
\hline Pulsed, $\tau=100 \mu \mathrm{s}, 1 \mathrm{kHz}$ & 283 & 409 \\
\hline Pulsed, $\tau=1000 \mu \mathrm{s}, 100 \mathrm{~Hz}$ & 313 & 436 \\
\hline Pulsed, $\tau=100 \mathrm{~ms}, 1 \mathrm{~Hz}$ & 428 & 491 \\
\hline $\mathrm{cW}$ & 384 & 454 \\
\hline
\end{tabular}

\subsection{Dynamic Thermal Impedance, $Z_{t h}$}

The high duty-cycle capability of different packages can be understood and compared numerically using the dynamic thermal impedance, $Z_{\mathrm{th}}$. Here, the temperature of the critical light-generating or active region $T_{\mathrm{AR}}$ (normally one to two quantum wells, for sources emitting at $\lambda=940 \mathrm{~nm}$ ) is tracked as a function of supplied heat, $P_{\text {therm }}$ and pulse width $\tau_{\mathrm{p}} . T_{\mathrm{AR}}$ is measured using the (time-averaged) centroid emission wavelength, which typically acts as a good thermometer $(\lambda$ increases monotonically and reproducibly with $T_{\mathrm{AR}}$, at around $0.38 \mathrm{~nm} / \mathrm{K}$ ), and $P_{\text {therm }}$ is obtained from measured applied current $I$, bias voltage $U$ (measured directly across the diode laser in 4-terminal configuration) and peak ( average in-pulse) power $P_{\text {opt }}$ via $P_{\text {therm }}=I U-P_{\text {opt }}$. This links the average heating, which regulates lifetime, solid angle (and hence $B$ ), $\lambda$ and spectral width $\lambda$ (hotter bars have wider spectra) with the operating point, for various operating conditions. Typically, $Z_{\text {th }}$ is a constant for a given $\tau_{p}, f$ and packaging configuration, obtained using a linear fit to plots of $T_{\mathrm{AR}}$ as a function of $P_{\text {therm. }}$. Figure 4 shows a comparison of $Z_{t h}$, measured in [10] for a rear-edge cooled commercial QCW stack and for the FBH design. In both cases, the values are calculated for HEC-DL sources (stacks) that are specifically designed for high duty cycle operation. $Z_{\text {th }}$ is in general a complex function of $\tau_{p}$ and $f$ with different time constants $\tau_{i}$ for different parts of the thermal path [21]. Here, the measurement data in Figure 4 was fitted using a simplified function with one thermal time constant $\tau$, which then allows $Z_{t h}$ to be extrapolated for various pulse widths $\tau_{p}$ as discussed in [22] and reprinted here for clarity, Equation (1), where $A$ is the saturation (cw) value for $Z_{\text {th }}$ and $y_{0}$ is an offset. Microchannel cooler performance is not shown here: $Z_{\text {th }}$ is low and relatively insensitive to variations in $\tau_{\mathrm{p}}$ and $f$.

$$
Z_{t h}=A\left(1+y_{0} \cdot e^{(-t / \tau)}\right)
$$

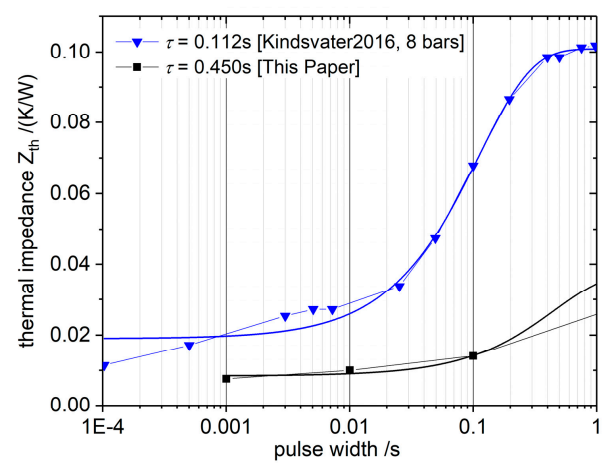

Figure 4. Dynamic thermal impedance for a commercially available pump module for various pulse conditions and the FBH stack module both measured at 10\% duty cycle. For the FBH design, the data point outside the graph is for $\mathrm{cw}$ operation. Time constants $\tau$ is taken from a fit function calculated following [22]. 
As one can see in Figure 4 the edge cooled mounting design used here (FBH concept) leads to $2 \ldots 3 \times$ lower $Z_{t h}$ than for the high duty-cycle optimized QCW bar design from [10] (e.g., saturation (cw) value for FBH: $0.038 \mathrm{~K} / \mathrm{W},[10]: 0.1 \mathrm{~K} / \mathrm{W}$, value at $1 \mathrm{~ms}$ : for $\mathrm{FBH}: 0.01 \mathrm{~K} / \mathrm{W},[10]: 0.025 \mathrm{~K} / \mathrm{W}$,) and longer saturation time (FBH: $\tau=450 \mathrm{~ms}$, [10]: $\tau=112 \mathrm{~ms}$ ). The time constant $\tau$ is obtained following [22], using the fit function given in Equation (1). Please note that higher $\tau$ leads to the module being less sensitive to rapid temperature changes, and hence more suitable for long-pulse operation. In addition the 1-cm bars in [10] are limited by the high duty cycle and high $Z_{\text {th }}$ values to operate with ca. $P_{\mathrm{opt}}=105 \mathrm{~W}$ per bar thus $P_{\mathrm{opt}}=105 \mathrm{~W}$ per $1 \mathrm{~cm}$ lateral aperture. In contrast, the FBH stack elements operate with ca. $P_{\text {opt }}=120 \mathrm{~W}$ from a $1200 \mu \mathrm{m}$ lateral aperture, which is ca. 10 times higher brightness than in [10].

\section{Use of High Duty Cycle Pumps for System Development and Pumping Trials}

\subsection{Example 1: Diode Pumped 100 ... 200 Hz Yb:YAG Disk Lasers (Max Born Institute, MBI)}

The high repetition rate pump sources presented here (and those from earlier generations), were used as part of the development of diode pumped solid state laser systems at the Max Born Institute (MBI), that use thin-disk Yb:YAG lasers to deliver pulses with $f=100 \ldots 200 \mathrm{~Hz}, \tau_{\mathrm{p}}=1 \mathrm{~ms}$ and a pulse energy in the Joule range. All systems were based on stacks of edge-cooled large area, long resonator emitters, and have been in long term use without failure in research laser systems.

In early work, diode laser mini arrays with $1.64 \mathrm{~mm}$ aperture and $4 \mathrm{~mm}$ resonator length that operated with $P_{\text {opt }}=100 \mathrm{~W}$ were used to construct stacks of 12 diode lasers. The emission from single stacks was optically combined and coupled into a fiber with $1.2 \mathrm{~mm}$ core and NA $=0.22$, to produce pump modules that delivered a peak power from fiber of $1 \mathrm{~kW}(1 \mathrm{~J}$ pulse energy in a pulse of $1 \mathrm{~ms}$ duration) at a duty cycle of $10 \%(f=100 \mathrm{~Hz})$ [23]. With the help of these early diode laser pump modules, the MBI was able to develop several laser systems consisting of different amplifiers based on Yb:YAG thin-disk technology [24], for example designed as driver for a laser-plasma based X-ray laser [25]. The first type of amplifier within this project was a regenerative amplifier using two of the 1 J pump modules to pump the Yb:YAG thin-disk, with a system schematic shown in Figure 5 left. Since this regenerative amplifier operates in a very stable manner, see Figure 5 right, the MBI has set up several of these systems. They are used on a routine basis with pulse energies between 200 and $300 \mathrm{~mJ}$ and reach a stability better than $0.3 \%$ (rms) [26]. The first system has operated since 2008 using $2 \times 1 \mathrm{~kW}$ pump diodes modules and is still in routine use as a driver for an XUV plasma source. The same two pump modules have been used ever since. To date, the total on-time of these diodes sums up to $7800 \mathrm{~h}$, without degradation.
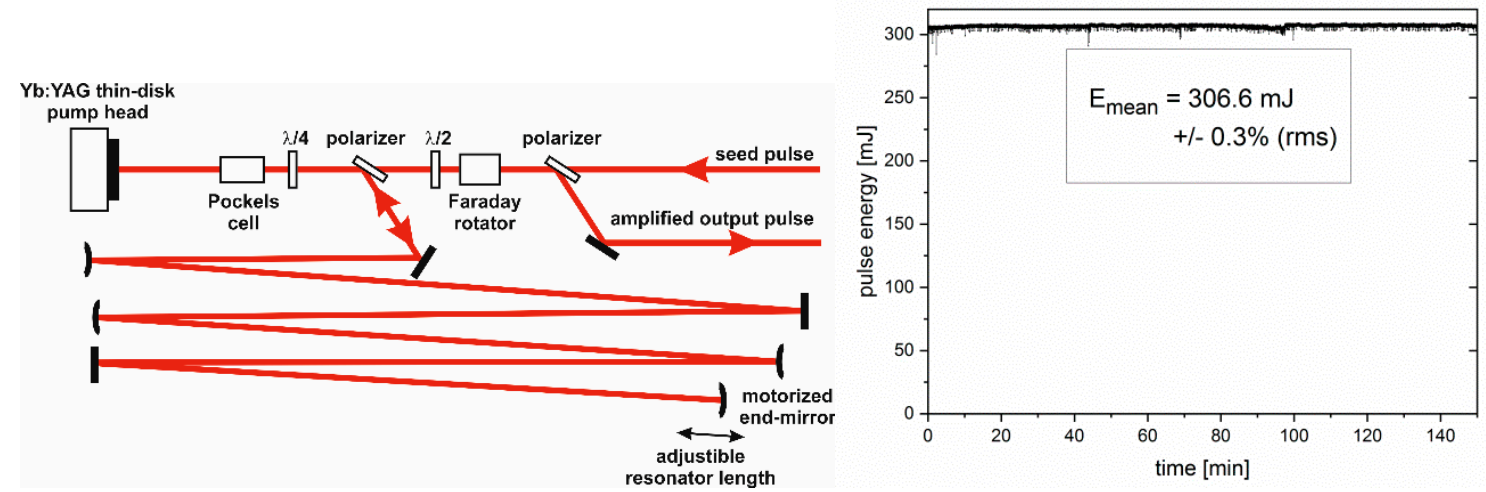

Figure 5. (Left) Sketch of the regenerative thin-disk amplifier. (Right) Pulse energy as function of time during normal operation. Output pulses with energy of more than $300 \mathrm{~mJ}$ and a fluctuation below $0.3 \%$ (rms) measured over $2.5 \mathrm{~h}$ were demonstrated. 
The most recent module designs, reviewed here, use two stacks of 28 diode lasers, each with $6 \mathrm{~mm}$ resonator and $1.2 \mathrm{~mm}$ aperture and $P_{\mathrm{opt}}=120 \mathrm{~W}$, for increased peak power from fibre of $6 \mathrm{~kW}$ $(1.9 \mathrm{~mm}$ core, $\mathrm{NA}=0.22)$ and duty cycle up to $20 \%\left(\tau_{\mathrm{p}}=1 \mathrm{~ms}, f=100 \ldots 200 \mathrm{~Hz}\right)$. In $2013 / 2014$, the FBH delivered six such pump diode modules to the MBI and two of these modules were used as a $12 \mathrm{~kW}$ pump source for Yb:YAG thin disks in a ring amplifier configuration, as shown in Figure 6 left. This ring amplifier implements two round trips. Seeded with $300 \mathrm{~mJ}$ from a regenerative amplifier, an output pulse energy of $1 \mathrm{~J}$ has been demonstrated $[27,28]$. Since saturation did not occur at this energy level, see Figure 6 right, the output energy could potentially further be increased by using stronger seed-pulses.
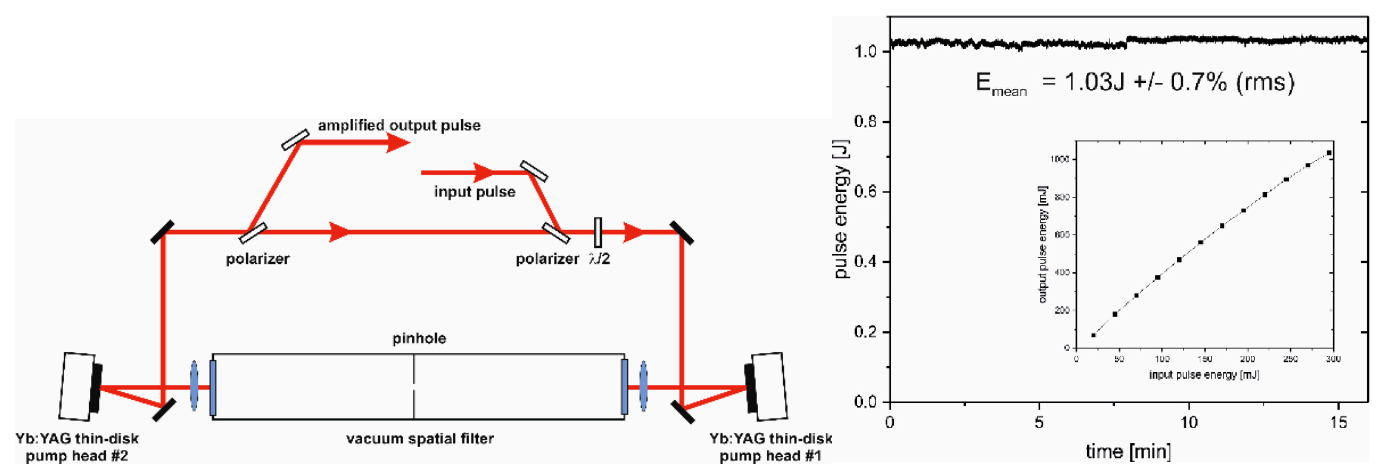

Figure 6. Sketch of the $1 \mathrm{~J}$ ring amplifier. Pulse energies of more than $1 \mathrm{~J}$ at $100 \mathrm{~Hz}$ repetition rate were demonstrated using two $6 \mathrm{~kW}$ pump diode modules at each amplifier head.

The $6 \mathrm{~kW}$ modules are also used on a daily basis with MBI's regenerative amplifier. In the standard operational mode, these amplifiers are pumped with $100 \ldots 200 \mathrm{~Hz}$ repetition rate and $1 \mathrm{~ms}$ pump pulse duration. Also, other operational modes were tested, maintaining the maximum duty cycle of $20 \%$ compatible with the pump module cooling system. A particularly interesting result was the increase of the repetition rate to $800 \mathrm{~Hz}$. This was accomplished by reducing the pulse duration of the pump diodes to $0.25 \mathrm{~ms}$. In this mode, the regenerative amplifier emitted laser pulses of more than $50 \mathrm{~mJ}$ energy at $800 \mathrm{~Hz}$ repetition rate. To date, the on-time of the $6 \mathrm{~kW}$ modules sums up to more than $3500 \mathrm{~h}$. There has been no measurable degradation in the output power of the pump sources. Overall, the diode laser pump sources discussed here have been successfully used in the development of several novel high repetition rate Joule-class laser systems, with reliable operation confirmed.

\subsection{Example 2: Initial Studies into High-Repetition Rate Pumping of Cryogenically Cooled Yb:YAG Disk Amplifiers (Institute for Optics and Quantum Electronics, IOQ Jena)}

The DIOPLE and DIPOLE100 developments showed that high energy class diode lasers used to pump solid state lasers can be successfully realised using cryogenically cooled Yb:YAG amplifiers, for pulse energies in the $10 \ldots 100 \mathrm{~J}$ range, at a repetition rate of $f=10 \mathrm{~Hz}$ [2-4]. Future systems, for example those studied within EuPRAXIA, need to scale repetition rates in these multi-Joule systems to $f=100 \mathrm{~Hz}$ and above. However, there are relatively few studies into the impact of high repetition rate on the characteristics of cryogenically cooled amplifier crystals. Therefore, in cooperation with the Institute for Optics and Quantum Electronics (IOQ) at the University of Jena and FBH, the pump modules reviewed here were used in a first assessment of $f=100 \mathrm{~Hz}$ pumping of an experimental cryogenically cooled disk amplifier. The experiments were performed under conditions that closely emulate EuPRAXIA use (for one possible amplifier design), but at lower total power $\left(P_{\text {opt }}=4 \mathrm{~kW}\right.$ instead of $300 \ldots 1200 \mathrm{~kW}$ ). In a first step, the thermal profile of a cryogenically cooled amplifier disk was measured with $f=100 \mathrm{~Hz}$ pumping, in an extension to the studies previously reported in [29]. In addition to the higher repetition rate pumping, a more advanced cooling system for the $Y b: Y A G$ disk was also used as reported in [29]. Specifically, the Yb:YAG amplifier disk (50 mm radius, 3 at. 
$\%$, comparable to [29]) was placed between two sapphire disks which were connected to a $\mathrm{LN}_{2}$ bath, see Figure 7. A gap between Yb:YAG and sapphire of $\sim 15 \mu \mathrm{m}$ or less was used, which leads to face cooling of the disk amplifier, for significantly improved heat extraction. The disk/sapphire assembly was located in a gas chamber which was filled with 1 bar $\mathrm{N}_{2}$ in order to establish good thermal contact between the amplifier and sapphire disks. Subsequently, the whole setup was placed in a vacuum chamber to ensure proper thermal insulation. A high power beam from a single diode laser pump source was centred onto the disk, with operating condition of $B=0.8 \mathrm{MW} / \mathrm{cm}^{2} / \mathrm{sr}\left(P_{\text {opt }}=3.8 \mathrm{~kW}\right)$, $f=100 \mathrm{~Hz}, \tau_{\mathrm{p}}=1 \mathrm{~ms}$, where $\lambda_{\mathrm{c}}=940 \mathrm{~nm}$ and $\Delta \lambda_{95 \%}=7 \mathrm{~nm}$ selected. The beam was imaged onto the disk with a spot diameter of $\sim 5 \mathrm{~mm}$. A second low power diagnostic laser (emitting at $967 \mathrm{~nm}$ ) was used to enable monitoring of the local temperature of the disk via changes in the intensity of the monitor beam, following [16,29]. The monitor laser illuminated the whole amplifier disk and the monitor intensity was detected with a camera placed behind the chamber. A standard optic was used to image the plane of the amplifier disk onto the camera focal plane. A chopper spinning with $f=100 \mathrm{~Hz}$ alternated the two laser beams blocking the high power pump light while taking an image of the monitor. The monitor intensity detected by the camera can be used as a thermometer because the absorption cross-section of the gain material depends on its temperature, following the same calibrated technique as in previous IOQ studies. Specifically, the ratio of the image intensity (dark signal to measurement) is proportional to the temperature of the gain media and is used to calculate the temperature gradient $[16,29]$.
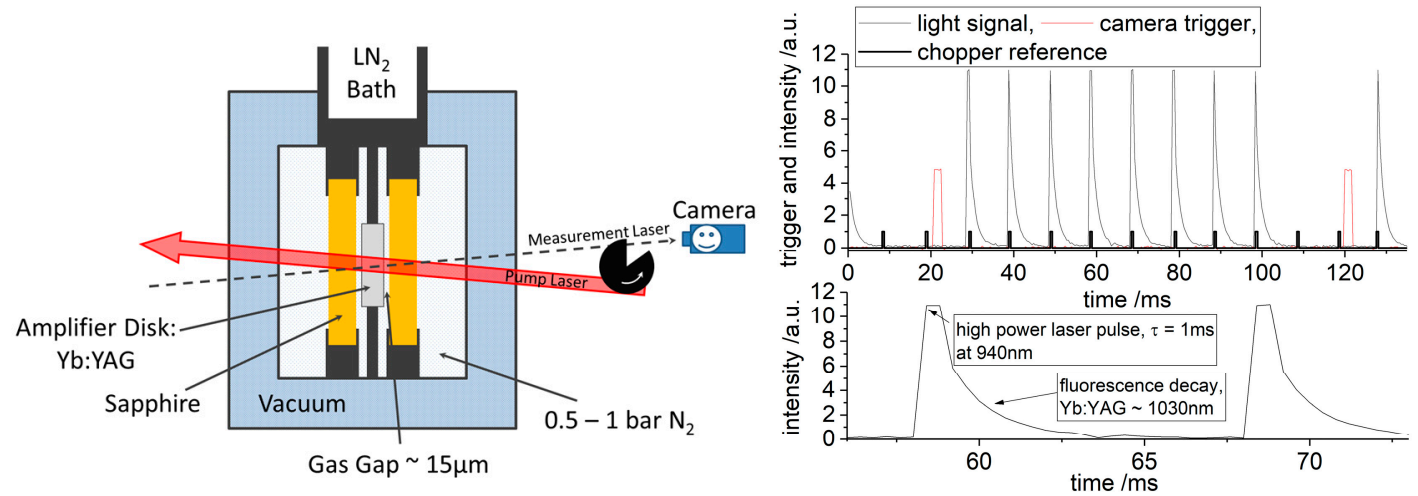

Figure 7. Left: Schematic depiction of the experimental setup for $100 \mathrm{~Hz}$ pumping trials of a cryogenically cooled Yb:YAG disk amplifier. The trigger wheel blocks and opens alternately the pump laser and the low power diagnostic laser. Right: Applied trigger regime and detected light pulse signal. The camera not only detects the pump light but also the florescence light from the amplifier disk and, therefore, detection of pump light on the camera has to be prevented.

The trigger regime is shown in Figure 7. The chopper provides the master trigger pulse at $f=100 \mathrm{~Hz}$, and a pulse generator provides a phase-stable $f=10 \mathrm{~Hz}$ trigger signal for the monitor signal, to match the $f=10 \mathrm{~Hz}$ frame limit of the camera.

The pulse conditions were further refined to enable an error-free measurement of temperature. The high power pump laser pulse initialised fluorescence in the Yb:YAG crystal which has a 1/e decay time (fluorescence lifetime) of $950 \mu \mathrm{s}$. Although this is much shorter than the $10 \mathrm{~ms}$ pulse-to-pulse spacing, the fluorescence is intense enough for sufficient light to disturb the temperature measurement would also be detected by the (highly sensitive) camera after one $10 \mathrm{~ms}$ period. Therefore, the trigger pulse generator was configured to provide a pulse train with eight trigger events (pump beam on) followed by two trigger events pause (pump beam off), see Figure 7 right. The pause of $20 \mathrm{~ms}$ is sufficient long to exclude the florescence light from the camera measurement and hence allow accurate temperature values to be obtained. We note that although pulse-pump spacing was equivalent to that for $f=100 \mathrm{~Hz}$ (period of $10 \mathrm{~ms}$ ), the two-trigger event pause applied for diagnostics meant that the effective total pump frequency was just $f=80 \mathrm{~Hz}$. The pump laser was operated for $30 \mathrm{~s}$, starting $5 \mathrm{~s}$ 
after the temperature monitoring began. The resulting measurement of temperature profile and local temperature increase is shown in Figure 8. The 2D temperature profile $25 \mathrm{~s}$ after start of measurement (20 s after start of pumping) is shown in Figure 8 and indicates a reasonably well-centred pump beam. Figure 8 also shows the time evolution of the temperature gradient of the disk (peak-edge). The temperature rises and reaches its maximum of approximately $125 \mathrm{~K}$ at $35 \mathrm{~s}$ after the start of experiment.
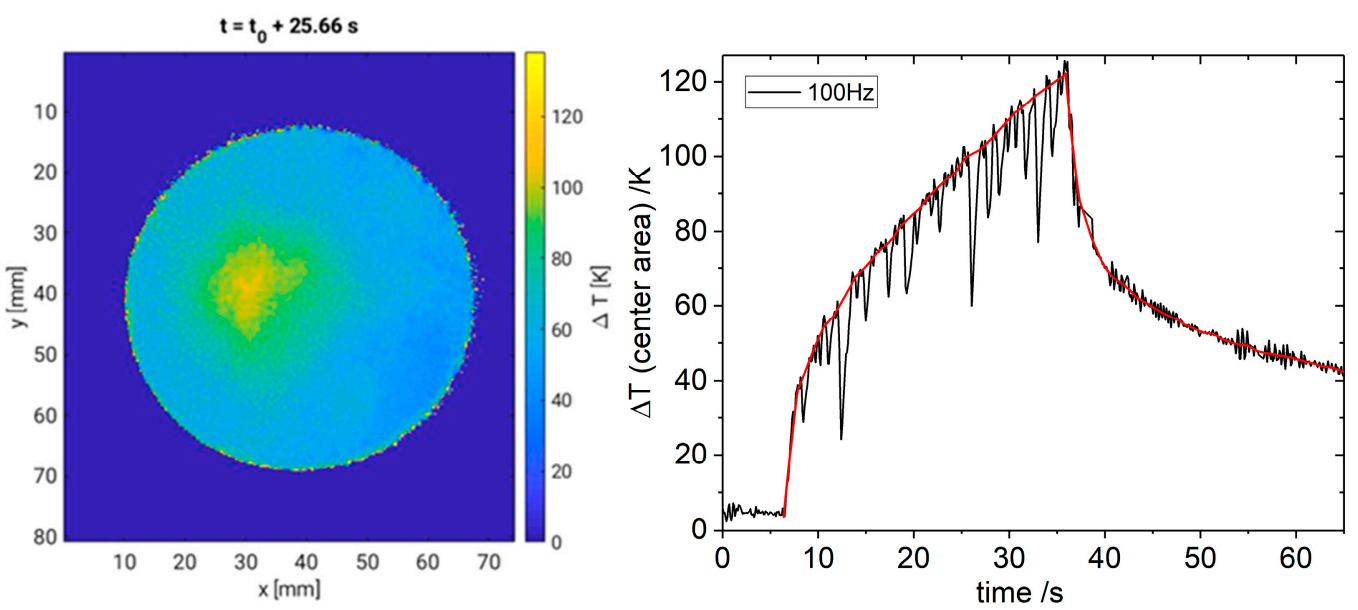

Figure 8. Evolution of temperature of a 3\% at. Yb:YAG disk amplifier, pumped with $B=0.8 \mathrm{MW} / \mathrm{cm}^{2} / \mathrm{sr}$ at $f=100 \mathrm{~Hz}$ and $\tau_{p}=1 \mathrm{~ms}$, in a cryogenically $\left(\mathrm{LN}_{2}\right)$ face-cooled configuration. Left: Temperature as function of position (false color, linearly scaled) for the Yb:YAG disk $25 \mathrm{~s}$ after measurements started (20 s after pumping begins). Right: Peak temperature increase over reference $\left(\mathrm{LN}_{2}\right)$ plane as function of time for pump pulses with $f=100 \mathrm{~Hz}$ (red curve: with measurement artefacts removed).

However, when the laser was switched off, the temperature should relax towards the cold head (70 K) temperature. Instead, the temperature relaxes to a high intermediate value before drifting back to the start value with a long time constant. The high temperatures are due to a general heating up of the whole laser head due to non-idealities in the thermal bond to the sapphire disks, which then leads to unrepresentatively high absolute temperatures developing. A more powerful cryogenic cooler or improved thermal joint would eliminate this problem, but this was not available at the time of the experiment. If the long time-constant overall heating can be eliminated, the authors estimate that a total in-crystal heating of around $20 \mathrm{~K}$ should be possible in this face cooled configuration under the same pump conditions, but this must be confirmed in future work. It should also be noted that the spikes in the pulse-on time shown in Figure 8 right are due to instabilities in the chopper reference signal, do not reflect fluctuations in the high power laser module, and can be addressed with an improved chopper in future experiments for improved signal quality. Overall, this initial pump experiments showed that when a repetition rate of $f=100 \mathrm{~Hz}$ is used, high temperatures can develop rapidly, even when an advanced cooling scheme is used. The experiments also showed the promise of the face-cooling approach, which extrapolates low temperatures, provided sufficiently effective overall heat extraction is available. Further studies of the thermal behaviour of and system design for cryogenically cooled amplifier crystals under high repetition rate pumping are clearly needed.

\section{Conclusions}

An overview of the state of the art in high power, high repetition rate diode laser pumps for high-energy class systems was presented. It was shown that commercial sources are currently either designed for low duty cycle operation or require microchannel coolers, which are not preferred for large systems on price and reliability grounds. Alternative high repetition rate pump modules developed at the FBH were shown to offer a viable path to fulfil the performance targets for high energy class systems without need for microchannel coolers, but currently at an overall power level $(6 \mathrm{~kW})$ lower than needed for large systems such as EuPRAXIA (>300 kW). The novel design of the FBH modules 
leads to at least $2 \ldots 3 \times$ lower thermal resistance than for conventional bar-based packaging and a longer thermal time constant of $\sim 450 \mathrm{~ms}$ ( $\sim 115 \mathrm{~ms}$ for bar-based designs), which means that a wide variety of pulse widths $(100 \mu \mathrm{s} \ldots 100 \mathrm{~ms})$ and duty cycles $(0-20 \%)$ can be addressed without excessive overheating or performance degradation. Results were also presented on the use of these high repetition rate pump modules in novel Joule-class pulsed laser systems based on room temperature Yb:YAG disk amplifiers, developed at the MBI. In addition, data from initial studies at IOQ Jena into the impact of high repetition rate pumping on cryogenically cooled Yb:YAG disk amplifiers was also presented. Further studies are needed in high repetition rate pumping, including further development of the diode laser pump sources.

Author Contributions: Conceptualization, P.C., I.W., J.K. and J.H.; software, M.H., B.E. and J.K.; validation, M.H., J.R., M.L., A.G. and J.K.; formal analysis, M.H., J.T., B.E., P.C. and J.K.; writing-original draft preparation, M.H., I.W. and P.C.; writing-review and editing, M.H., P.C., J.K., I.W. and J.H.; visualization, M.H. and P.C.; supervision, P.C.; project administration, P.C., J.H. and I.W.; funding acquisition, P.C.

Funding: This work was supported in part by the Senate Competition Committee of the Leibniz Association within the Joint Initiative for Research and Innovation of the German Federal Government and the Regions. This work was also funded in part via the European Regional Development Fund (ERDF) under contract number 20072013 2/42. We thank DESY and EuPRAXIA consortium for supporting the cooperative measurements between FBH and IOQ Jena.

Acknowledgments: We would like to thank Trumpf Laser Berlin (C2Go) for supporting the assembly of the stack modules and Protech Berlin $\mathrm{GmbH}$ for the fabrication of the customized fiber."

Conflicts of Interest: The authors declare no conflict of interest.

\section{References}

1. Assmann, R.; Specka, A. EuPRAXIA - A European Plasma Research Accelerator with Excellence in Applications. Available online: https:/indico.cern.ch/event/489461/contributions/2168493/attachments/ 1305881/1951582/2016-07-01_EuPRAXIA-WP1-assmann-out.pdf (accessed on 3 July 2019).

2. Mason, P.D.; Banerjee, S.; Ertel, K.; Phillips, P.J.; Butcher, T.J.; Smith, J.M.; De Vido, M.; Tomlinson, S.; Chekhlov, O.; Shaikh, W.; et al. DiPOLE100: A 100J, 10Hz DPSSL using cryogenic gas cooled Yb:YAG multi slab amplifier technology. Proc. SPIE 2015, 9513, 951302. [CrossRef]

3. De Vido, M.; Ertel, K.; Mason, P.D.; Banerjee, S.; Phillips, P.J.; Smith, J.M.; Butcher, T.J.; Chekhlov, O.; Divoky, M.; Pilar, J.; et al. A 100J-level nanosecond pulsed DPSSL for pumping high-efficiency, high-repetition rate PW-class lasers. Proc. SPIE 2017, 10082, 100820M. [CrossRef]

4. Butcher, T.; Mason, P.; Banerjee, S.; Ertel, K.; Phillips, P.J.; Smith, J.; De Vido, M.; Chekhlov, O.; Divoky, M.; Pilat, J.; et al. A 100 J-level nanosecond DPSSL for high energy density experiments. Proc. SPIE 2017, 10238, 1023802. [CrossRef]

5. Wessling, C.; Rübenach, O.; Hambücker, S.; Sinhoff, V.; Banerjeea, S.; Ertel, K.; Mason, P. Efficient pumping of inertial fusion energy lasers. Proc. SPIE 2013, 8602, 86020I. [CrossRef]

6. Bonati, G. Prospects for the Diode Laser Market. Laser Tech. J. 2010, 7, 37-40. [CrossRef]

7. Fassbender, W.; Kissel, H.; Koenning, T.; Patterson, S.; Biesenbach, J. Reliable QCW diode laser arrays for operation with high duty cycles. Proc. SPIE 2017, 10085, 1008509. [CrossRef]

8. Wölz, M.; Pietrzak, A.; Kindsvater, A.; Meusel, J.; Stolberg, K.; Hülsewede, R.; Sebastian, J.; Loyo-Maldonado, V. Laser diode stacks: Pulsed light power for nuclear fusion. High Power Laser Sci. Eng. 2016, 4, e14. [CrossRef]

9. Zhang, P.; Liu, X.; Zhu, Q.; Wang, J. Thermal characteristics of compact conduction-cooled high power diode laser array packages. Proc. SPIE 2017, 10085, 100850A. [CrossRef]

10. Kindsvater, A.; Schröder, M.; Werner, E.; Seidel, S.; Wölz, M.; Loyo-Maldonado, V. High duty-cycle, high-efficiency QCW stacks for medical applications. Proc. SPIE 2016, 9733, 97330M. [CrossRef]

11. Platz, R.; Eppich, B.; Rieprich, J.; Pittroff, W.; Erbert, G.; Crump, P. High duty cycle, highly efficient fiber coupled 940-nm pump module for high-energy solid-state lasers. High Power Laser Sci. Eng. 2016, 4, e3. [CrossRef] 
12. Platz, R.; Frevert, C.; Eppich, B.; Rieprich, J.; Ginolas, A.; Kreutzmann, S.; Knigge, S.; Erbert, G.; Crump, P. Progress in high duty cycle, highly efficient fiber coupled 940-nm pump modules for high-energy class solid-state lasers. Proc. SPIE 2018, 10513, 1051319. [CrossRef]

13. Crump, P.; Karow, M.M.; Knigge, S.; Maasdorf, A.; Traenkle, G.; Lotz, J.; Fassbender, W.; Neukum, J.; Körner, J.; Boedefeld, R.; et al. Progress in joule-class diode laser bars and high brightness modules for application in long-pulse pumping of solid state amplifiers. Proc. SPIE 2017, 10086, 100860E. [CrossRef]

14. Pittroff, W.; Eppich, B.; Erbert, G.; Platz, R.; Tyralla, D.; Tränkle, G. Simple design for fiber coupled 9xx nm kW-QCW pump module with high duty cycle based on customized chips and lateral heat removal. Proc. SPIE 2014, 8965, 896515. [CrossRef]

15. Negoita, V.C.; Vethake, T.; Jiang, J.; Roff, R.; Shih, M.; Duck, R.; Bauer, M.; Mite, R.; Boucke, K.M.; Treusch, G. High peak power diode stacks for high energy lasers. Proc. SPIE 2015, 9345, 934504. [CrossRef]

16. Körner, J.; Jambunathan, V.; Hein, J.; Seifert, R.; Loeser, M.; Siebold, M.; Schramm, U.; Sikocinski, P.; Lucianetti, A.; Mocek, T.; et al. Spectroscopic characterization of $\mathrm{Yb}^{3+}$-doped laser materials at cryogenic temperatures. Appl. Phys. B 2014, 116, 75. [CrossRef]

17. ISO 11146-2:2005. Lasers and laser-related equipment - Test methods for laser beam widths, divergence angles and beam propagation ratios - Part 2: General astigmatic beams. Available online: https://www.iso. org/standard/33626.html (accessed on 3 July 2019).

18. Winterfeldt, M.; Crump, P.; Knigge, S.; Maaßdorf, A.; Zeimer, U.; Erbert, G. High Beam Quality in Broad Area Lasers via Suppression of Lateral Carrier Accumulation. IEEE Photonics Technol. Lett. 2015, $27,1809$. [CrossRef]

19. Winterfeldt, M.; Crump, P.; Wenzel, H.; Erbert, G.; Tränkle, G. Experimental investigation of factors limiting slow axis beam quality in 9xx $\mathrm{nm}$ high power broad area diode lasers. J. Appl. Phys. 2014, 116, 063103. [CrossRef]

20. Huber, M.; Saupe, F.; Wuest, P.; Forrer, H.; Kunz, A.; Moser, H. Impact of absorption in fast axis collimation lenses. Proc. SPIE 2018, 10513, 105130P. [CrossRef]

21. Liu, X.; Hu, M.H.; Caneau, C.G.; Bhat, R.; Zah, C.-E. Thermal management strategies for high power semiconductor pump lasers. IEEE Trans. Compon. Packag. Technol. 2006, 29, 268. [CrossRef]

22. Székely, V. A new evaluation method of thermal transient measurement results. Microelectron. J. 1997, $28,277$. [CrossRef]

23. Pittroff, W.; Erbert, G.; Beister, G.; Bugge, F.; Klein, A.; Knauer, A.; Maege, J.; Ressel, P.; Sebastian, J.; Staske, R.; et al. Mounting of high power laser diodes on boron nitride heat sinks using an optimized Au/Sn metallurgy. IEEE Trans. Adv. Packag. 2001, 24, 434. [CrossRef]

24. Tümmler, J.; Jung, R.; Stiel, H.; Nickles, P.V.; Sandner, W. High-repetition-rate chirped-pulse-amplification thin-disk laser system with joule-level pulse energy. Opt. Lett. 2009, 34, 1378. [CrossRef]

25. Stiel, H.; Tümmler, J.; Jung, R.; Nickles, P.V.; Sandner, W. X-ray laser takes the $100 \mathrm{~Hz}$ barrier. Proc. SPIE 2009, 7451, 745109. [CrossRef]

26. Jung, R.; Tümmler, J.; Will, I. Regenerative thin-disk amplifier for $300 \mathrm{~mJ}$ pulse energy. Opt. Express 2016, 24, 883. [CrossRef] [PubMed]

27. Will, I.; Tümmler, J.; Nubbemeyer, T.; Jung, R.; Sandner, W. Vorrichtung zur Verstärkung von Gepulster Laserstrahlung mit Hoher Energie der Laserpulse und Hoher Mittlerer Leistung. German Patent No. DE102013208377, 7 May 2013.

28. Jung, R.; Tümmler, J.; Nubbemeyer, T.; Will, I. Two-Channel Thin-Disk Laser for High Pulse Energy. Proc. SPIE 2015, AW3A.7. [CrossRef]

29. Körner, J.; Yue, F.; Hein, J.; Kaluza, M.C. Spatially and temporally resolved temperature measurement in laser media. Opt. Lett. 2016, 41, 2525. [CrossRef]

(C) 2019 by the authors. Licensee MDPI, Basel, Switzerland. This article is an open access article distributed under the terms and conditions of the Creative Commons Attribution (CC BY) license (http://creativecommons.org/licenses/by/4.0/). 